\title{
Evaluation of Masticatory Performance and Patient Satisfaction Before and After Posterior Implant Restoration
}

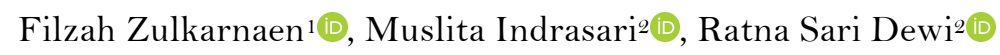

${ }^{1}$ Prosthodontics Residency Program, Faculty of Dentistry, Universitas Indonesia, Jakarta, Indonesia.

${ }^{2}$ Department of Prosthodontics, Faculty of Dentistry, Universitas Indonesia, Jakarta, Indonesia.

Correspondence: Ratna Sari Dewi, DDS, Department of Prosthodontics, Faculty of Dentistry, Universitas Indonesia, Jalan Salemba Raya No.4, Jakarta Pusat, Jakarta 10430, Indonesia. E-mail: ratnasaridewi.drg@gmail.com

Academic Editor: Alessandro Leite Cavalcanti

Received: 17 January 2020 / Review: 28 July 2020 / Accepted: 13 September 2020

How to cite: Zulkarnaen F, Indrasari M, Dewi RS. Evaluation of masticatory performance and patient satisfaction before
and after posterior implant restoration. Pesqui Bras Odontopediatria Clín Integr. 2021; $21: e 5894$.
https://doi.org/10.1590/pboci.2021.046

\begin{abstract}
Objective: To evaluate the improvement in masticatory performances two weeks after posterior implant restoration. Material and Methods: Nine patients with missing first and second molars were included in the study. Masticatory performances were evaluated using subjective and objective methods utilizing colorchangeable chewing gum and the visual analog scale (VAS) assessment. The subjects were asked to chew the color-changeable chewing gums before and two weeks after the posterior implant restoration. Their expectations regarding aesthetics and function were verified on the VAS before implant placement. Additionally, the VAS was used for the posttreatment completion rating two weeks after the implant restoration. Results: Significant differences in masticatory performance were noted at baseline (before implant posterior restoration) and two weeks after implant restoration $(\mathrm{p}<0.05)$. The posttreatment aesthetic and functional expectations ratings significantly exceeded the expectations $(\mathrm{p}<0.05)$. Conclusion: Masticatory performances were improved two weeks after implant restoration. In addition, the significant posttreatment ratings of the patients exceeded their initial expectations. In particular, patients with poor masticatory functions demonstrated significant improvements and satisfaction following implant restoration compared to those with good mastication. These findings indicate that a posterior implant restoration can increase the masticatory performance.
\end{abstract}

Keywords: Dental Implantation; Mastication; Chewing Gum; Patient Satisfaction. 


\section{Introduction}

With the increase in the average life span, people are becoming increasingly aware of maintaining the quality of life, including the dietary lifestyle [1]. Dental implants are considered as one of the most common and popular treatment options for edentulous patients, and remarkable advancements in the techniques and materials used in the field have been made [1,2]. It is known that dental implants have many advantages, such as superior masticatory efficiency and adjacent teeth preservation, compared to other prosthetics; however, high costs and the duration of the treatment are the main limitations of dental implant therapy [1].

Many patients are reluctant to undergo implant treatment due to the surgical procedure and are especially apprehensive if a bone augmentation procedure is required [1,3]. Therefore, clinicians and patients are looking for implant options that can reduce the procedure's invasiveness or increase the speed of osseointegration [1]. Owing to the outstanding mechanical and biological properties, Roxolid SLActive Implants (Institut Straumann AG, Basel Switzerland) offer more treatment options than many other implants by reducing invasiveness, preserving the bone, and minimizing the extent of bone grafting. Roxolid is a highperformance alloy specifically designed to offer higher strength than pure titanium. In principle, the SLA surface is produced by coarse grit-blasting with $0.25-0.5 \mathrm{~mm}$ corundum grit at 5 bar, followed by acid-etching. SLActive surfaces are produced with the same sandblasting and acid-etching technique; however, they are rinsed under nitrogen protection to prevent air exposure and then stored in a sealed glass tube containing isotonic sodium chloride $(\mathrm{NaCl})$ solution. Schwarz claimed that this specific preparation process leads to a hydroxylated/hydrated surface, which retains the high surface energy by reducing the adsorption of potential contaminants from the atmosphere (e.g., hydrocarbons and carbonates). The SLActive surface allows for fast and predictable osseointegration enabling a safer and faster treatment method with high treatment predictability [4].

In general, dental implant placement's success rate is assessed according to clinical parameters, such as stability and radiographic bone loss [5]. Other than osseointegration, there has been an increasing need for dentists to gather subjective and objective information about patients' masticatory performances before and after dental treatment [1,3]. However, patients often find the dental terminology difficult to understand [3]. From the patients' point of view, the prosthodontic treatment's success depends on their ability to chew well. Therefore, an evaluation of the changes in masticatory function is more appropriate than typical histological approaches [3,6].

Some previous studies have used alveolar bone loss or implant movement to evaluate implant success over a relatively long follow-up period, e.g., several years after implant restoration. However, most patients use their prosthodontic implants to chew food only a few days after undergoing implant surgery [3]. Recently, immediate placement and immediate loading have become popular in implant dentistry due to increased demand for time-saving procedures and immediate satisfaction [2,3,5]. Hence, it is important to evaluate the changes in masticatory performances over a short period [3]. Unfortunately, there is a lack of research on the subject and claims that mastication can be improved within a short period after implant restoration have not been thoroughly investigated. In addition, masticatory performances may be expected to differ according to the status or condition of the patients' remaining teeth, even if they have only one or two missing teeth [2,3].

A method suitable for the task can objectively and reproducibly evaluate the therapeutic goals concerning individual masticatory performance $[7,8]$. A measurement at the beginning of the treatment, a comparative measurement at the end, and additional measurements during the rehabilitation process can allow for an objective evaluation of the treatment outcome. Therefore, a method that combines high precision, 
practicability, and limited temporal or technical requirements is necessary. Apart from the described methods, a measurement technique through color-changeable chewing gum seems most likely to fulfill these requirements due to its simplicity and reported superior differentiability [8]. A Japanese research group has developed this method to allow for simple measurement of the masticatory performance through the change in the chewing gum's color. The material is specially developed for that purpose and is called the Masticatory Performance Evaluating Gum Xylitol (Lotte Co., Ltd., Tokyo, Japan) [7,8].

Other researches have evaluated the efficacy of dental implant treatment based on patient satisfaction, and in most of these studies, the patients stated that they were satisfied with the treatment $[1,9,10]$. Since patients who spend more money and time for their dental treatment expect satisfactory services and results, it is important to understand the factors that influence patient satisfaction to provide better services in the future. However, some factors could have a negative impact on patient satisfaction, as is the case of patients with neuroticism. Several studies have examined the satisfaction levels of implant patients by comparing the conditions before and after surgery. Besides, the evaluation of factors that influence patient satisfaction has been performed. In one study on pain-related factors, the visual analog scale (VAS) scores of more than $60 \%$ of patients during and after implant surgery were reported as "mild" to "moderate" in grade. Furthermore, Levi et al. stated that patients' expectations are another important factor that can influence the patient's satisfaction with the implant treatment [Q].

Therefore, the present study aimed to compare the masticatory performances among patients with two missing molars and compare their expectations before and two weeks after implant restoration, using both objective and subjective evaluation methods [2,3].

\section{Material and Methods}

Ethical Clearance

This study was approved by the ethical committee of the Faculty of Dentistry, Universitas Indonesia (13 /Ethical Approval /FKGUI/III/2019).

Sampling

Of 39 patients, nine patients were retrieved in September 2019 at the Dental Hospital Faculty of Dentistry, Universitas Indonesia. The inclusion criteria included patients aged between 20 and 60 years in the initial records, missing first and second molars, able to communicate well and cooperatively, can answer questionnaires without the help of others, not senile, and willing to be included in the study. Patients with fixed restorative materials (e.g., crowns or bridges) were considered to have normal teeth. The following patients were excluded: those who had abnormal mastication due to any oral pain, temporomandibular disorder, or severe malocclusion; had lost teeth or crowns due to severe caries; were pregnant, or had a severe systemic disease such as diabetes or cardiovascular disease.

\section{Clinical Procedure}

An implant fixture was placed at the site of the missing teeth, and the state of the implant-bone integration was examined in every patient prior to participation in the study. Each patient received a Roxolid SLAactive implant endosseous of 4.1 or $4.8 \mathrm{~mm}$ diameter made of TiZr alloy (Institut Straumann AG, Basel Switzerland). The implants were inserted according to each operator's normal standard operational protocol and based on the manufacturer's standard surgical guidelines. The dental implant treatment was completed by 
the residents of the Faculty of Dentistry, Universitas Indonesia. Surgeries for implant placement followed the same protocol in planning, antiseptic care, pre- and postoperative medication, and drilling sequences. The date of implant restoration and the edentulous period were different among the patients. No additional surgical procedures (e.g., sinus lifts, alveolar nerve lateralization, or bone grafts) were included in the study design.

One dentist examined each patient's mouth and maintained the examination records. Each patient filled out a self-assessed VAS form. The patients were asked to indicate their expectations about the aesthetic and functional results of their dental implant treatment, ranging from o (indicated by the expression "worst results") to 10 (indicated by the expression "best results") on a $10 \mathrm{~cm}$ scale in the VAS.

The specific questions asked before the treatment were: (1) On a scale of o to 10, how would you score the functional benefits you expect from the treatment (mastication, comfort, retention in the mouth)?; and (2) On a scale of o to 10, how would you score the aesthetic benefits you expect from the treatment? After completing the treatment, a week after the final adjustments (which were usually done during three appointments after installation of the prosthesis), they were again asked to indicate, on the same VAS, their ratings of the outcomes of the two aforementioned parameters by answering the following questions: (1) On a scale of o to 10, how would you score the functional benefits you observed from the treatment (mastication, comfort, retention in the mouth)?; and (2) On a scale of o to 10 , how would you score the aesthetic benefits you observed as a result of the treatment?

After completion of the assessment, the patients were asked to chew the color-changeable chewing gum (Masticatory Performance Evaluating Gum Xylitol; Lotte Co., Ltd., Tokyo, Japan) to measure the masticatory performance. All other implant materials, including abutments and prostheses, were installed onto the implant fixture in the patients' mouths. For the color change measurement, the chewing gum was placed between two polyethylene films and flattened to a thickness of $1.5 \mathrm{~mm}$ using two glass plates. The color analysis was performed by measuring the color differences before and after the mastication process. After chewing, the values were measured utilizing a colorimeter. A recall examination was performed two weeks later.

\section{Data Analysis}

Data were analysed using IBM SPSS Statistics Software, version 20 (IBM Corp., Armonk, NY, USA). Descriptive statistics were used to calculate relative frequencies. Non-parametric Wilcoxon test was used to check the technical error of measurement by comparing two paired groups with $\mathrm{p}<0.05$. Non-parametric Spearman test was used to measure the degree of association between two variables with $\mathrm{p}<0.05$.

\section{Results}

Nine patients (age range, 26-55 years) were enrolled; among them, four (44.4\%) were aged between 46 to 55 years old (Table 1). There was more female $(66.7 \%)$, and one patient $(31.7 \%)$ underwent two-implant placements simultaneously.

Table 1. Distribution of patients based on age, sex, and implant placement.

\begin{tabular}{|c|c|c|}
\hline Variables & $\mathbf{N}$ & $\%$ \\
\hline \multicolumn{3}{|l|}{ Age (Years) } \\
\hline $26-35$ & $\mathcal{Q}$ & 22.2 \\
\hline $36-45$ & 3 & 33.3 \\
\hline $46-55$ & 4 & 44.4 \\
\hline \multicolumn{3}{|l|}{ Gender } \\
\hline Male & 3 & 33.3 \\
\hline Female & 6 & 66.7 \\
\hline
\end{tabular}




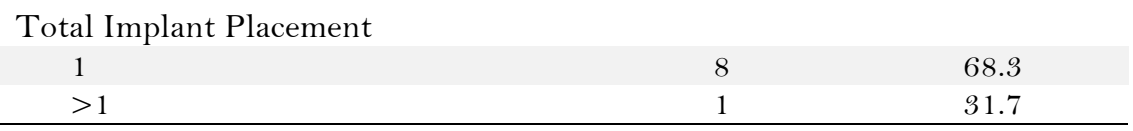

The indicator of masticatory performance (using the color-chewing gum method) showed significant increases after dental implant posterior restoration (before $=72.83 \pm 9.63$ vs. after $=82.98 \pm 6.41$ ), presumably due to recovery at the site of the missing molars $(\mathrm{p}<0.05)$.

A $10 \%$ increase in masticatory performance was observed two weeks after the implant restoration compared with the edentulous state before implant placement. The average VAS ratings for aesthetics and function before and after implant treatment are shown in Table 2. The outcome significantly exceeded the pretreatment expectations. In addition, positive correlations were found between the expectations and posttreatment ratings for aesthetic outcomes (Spearman's rho $=0.49 ; \mathrm{p}<0.05)$ and function (Spearman's rho $=$ $0.62, \mathrm{p}<0.05)$

Table 2. Comparison of average VAS patient pretreatment and posttreatment ratings.

\begin{tabular}{lccccc}
\hline Visual Analog Scale & \multicolumn{2}{c}{ Esthetics } & \multicolumn{2}{c}{ Function } & p-value* \\
& Before & After & Before & After & \\
\hline Average & 5.0 & 9.1 & 5.1 & 9.0 & $<0.05$ \\
Confidence Interval & 0.62 & 0.42 & 0.74 & 0.49 & $<0.05$ \\
\hline *Wilcoxon test.
\end{tabular}

\section{Discussion}

The reduction in masticatory function due to tooth loss can cause significant problems, including difficulties with food mixing and swallowing [2,3,6]. For patients with a missing tooth in the molar area, dental implants are considered the primary option [9]. However, the improvement in masticatory function in patients with implant restorations using subjective and objective masticatory evaluation methods has not been measured so far.

Existing studies have identified changes in masticatory performances before and after implant restoration in the molar area. In this study, a self-evaluation VAS assessment was used to measure the inconvenience associated with implant placement and to evaluate the aesthetic and functional posttreatment ratings of dental implant therapies in the patients [2]. In addition, color-changeable chewing gums were used to obtain quantitative analysis parameters of the various changes in masticatory function following implant restoration [3]. The color-changeable gum appears to be the easiest to use among the different methods available. Both techniques described in this review (visual matching with published color scales vs. colorimetric measurement) can be used to evaluate the masticatory performance in the color-changeable gum method. The evaluation should be based on color scales if the sample sizes are large enough to detect tiny differences [8].

We evaluated the changes in masticatory performance to detect the short-term improvements gained from an implant restoration. Two weeks is a relatively short period to evaluate the masticatory function, particularly when compared with previous studies that evaluated masticatory functions ranging from two weeks to five years after restoration with removable dental prostheses or partial implant fixed dental prostheses [3,6,11]. For several decades, dental implant restoration has been used to address the need for immediate functional and aesthetic rehabilitation in patients $[5,11]$. It is important for patients to experience immediate success. The majority of the previously conducted studies have evaluated the survival rate of the implant restoration after long periods. Kang et al. [3] reported that patients' oral health-related quality of life 
with implant prostheses increased rapidly. In keeping with these findings, the present study provides evidence for the short-term improvement in masticatory performance after implant restoration [3].

An interesting aspect is the wide range $(0-10)$ of posttreatment function ratings, which indicate that patients were highly sensitive to minor variations in prosthetic function. In a recent survey, Baracat et al. [Q] observed a contradictory result regarding gender-related attitudes about dental appearance. They assumed that Swedish women were more sensitive to dental appearance deficiencies; instead, they found that men emphasized the importance of dental appearance more than women [Q]. The common belief is that women are more interested in their appearance than men $[5,9,10,12]$.

Another result of the present study that deserves highlighting is that we found a positive correlation between pretreatment expectations and the final posttreatment ratings for both aesthetics and function, as Kang et al. [3] suggested. These correlations indicate that patients' expectations influence their satisfaction. It also emphasizes that the dental professional should assess patients' expectations to predict how they will evaluate their dentures after treatment [3,9]. Several important factors influencing patients' expectations before dental implant treatment and evaluation after treatment, such as previous prosthodontic experiences, personality traits, implant position, and other factors, were not evaluated in the present study [1,5,9-11]. We believe that further research involving larger numbers of patients and covering a wider range of variables should be conducted to increase our knowledge about the patients' pretreatment expectations and the final evaluation after dental implant therapies.

There were several limitations to the present study. Oral conditions such as caries, hypersensitivity, crack, and periodontitis, which could affect implant placement, were not considered in the exclusion criteria. In addition, we did not consider other restorative materials in the remaining dentition, although these could have affected the masticatory performance. Furthermore, the bite force is strongly related to mastication and oral conditions.

\section{Conclusion}

This study confirmed that masticatory performance, as evaluated subjectively using the VAS and objectively using the color-changeable chewing gum method, could increase two weeks after implant restoration. The enhancement in masticatory performance was particularly high in patients with poor mastication before the implant restoration. In addition, the patients' aesthetic and functional posttreatment ratings of dental implant therapies were significantly higher than their expectations before these therapies. A positive correlation was observed between the expectations and the posttreatment completion ratings for both aesthetics and function.

\section{Authors' Contributions}

\begin{tabular}{|c|c|c|}
\hline $\mathrm{FZ}$ & (D) https://orcid.org/0000-0001-8391-5238 & $\begin{array}{l}\text { Conceptualization, Methodology, Formal Analysis, Investigation, Writing - Original Draft and } \\
\text { Writing - Review and Editing. }\end{array}$ \\
\hline MI & (D) https://orcid.org/0000-0002-5138-285X & $\begin{array}{l}\text { Conceptualization, Methodology, Formal Analysis, Investigation, Writing - Original Draft and } \\
\text { Writing - Review and Editing. }\end{array}$ \\
\hline RSD & (D) https://orcid.org/0000-0002-8411-4755 & $\begin{array}{l}\text { Conceptualization, Methodology, Formal Analysis, Investigation, Writing - Original Draft and } \\
\text { Writing - Review and Editing. }\end{array}$ \\
\hline
\end{tabular}

\section{Financial Support}

Hibah Publikasi Internasional Terindeks Untuk Tugas Akhir Mahasiswa (PITTA) 2019 from Directorat Research and Community Engagement Universitas Indonesia. 


\section{Conflict of Interest}

The authors declare no conflicts of interest.

\section{Data Availability}

The data used to support the findings of this study can be made available upon request to the corresponding author.

\section{References}

[1] Kim Y, Kim H, Yi Y, Yun P. Evaluation of subjective satisfaction of dental implant patients. J Korean Assoc Oral Maxillofac Surg. 2014; 4O(3):130-4. https://doi.org/10.5125/jkaoms.2014.40.3.130

[2] Baracat LF, Teixeira AM, Santos MBF, Cunha VPP, Marchini L. Patients' expectations before and evaluation after dental implant therapy. Clin Implant Dent Relat Res 2011; 13(2):141-5. https://doi.org/10.1111/j.1708-8208.2009.00191.x.

[3] Kang S, Lee S, Kwon H, Kim B, Kim B. Short-term improvement of masticatory function after implant restoration. J Periodontal Implant Sci 2015; 45(6):205-9. https://doi.org/10.5051/jpis.2015.45.6.205

[4] Wennerberg A, Galli S, Albrektsson T. Current knowledge about the hydrophilic and nanostructured SLActive surface. Clin Cosmet Investig Dent 2011; 3:59-67. https://doi.org/10.2 147/CCIDEN.S15949

[5] Youk S, Lee J, Park J, Heo S, Roh H, Park E. A survey of the satisfaction of patients who have undergone implant surgery with and without employing a computer-guided implant surgical template. J Adv Prosthodont 2014; 6(5):395-405. https://doi.org/10.4047/jap.2014.6.5.395

[6] Gonçalves TMSV, Campos CH, Gonçalves GM, Moraes M, Garcia RCMR. Mastication improvement after partial implant-supported prosthesis use. J Dent Res. 2013; $92(12 \quad$ Suppl):189S-94S. https://doi.org/10.1177/0022034513508556

[7] Hama Y, Kanazawa M, Minakuchi S, Uchida T, Sasaki Y. Reliability and validity of a quantitative color scale to evaluate masticatory performance using color-changeable chewing gum. J Med Dent Sci 2014; 61(1):1-6.

[8] Tarkowska A, Katzer L, Ahlers MO. Assessment of masticatory performance by means of a color-changeable chewing gum. J Prosthodont Res 2017; 61(1):9-19. https://doi.org/10.1016/j.jpor.2016.04.004

[9] $\mathrm{Su}$ O. A study on satisfaction of dental implant patients. Available from: https://scholarcommons.sc.edu/cgi/viewcontent.cgi?article=5425\&context=etd. [Accessed on January 10, 2020).

[10] Annibali S, Vestri AR, Pilotto A, Monaca GL, Carlo SD, Cristalli MP. Patient satisfaction with oral implant rehabilitation: evaluation of responses to a questionnaire. Ann Stomatol 2010; 1(3-4):2-8.

[11] Vieira RA, Melo ACM, Budel LA, Gama JC, de Mattias Sartori IAM, Thomé G. Benefits of rehabilitation with implants in masticatory function: is patient perception of change in accordance. J Oral Implantol 2014; 4O(3):263-9. https://doi.org/10.1563/AAID-JOI-D-1 1-00208 